\title{
Groups not presentable by products
}

\author{
D. Kotschick and C. Löh*
}

\begin{abstract}
In this paper we study obstructions to presentability by products for finitely generated groups. Along the way we develop both the concept of acentral subgroups, and the relations between presentability by products on the one hand, and certain geometric and measure or orbit equivalence invariants of groups on the other. This leads to many new examples of groups not presentable by products, including all groups with infinitely many ends, the (outer) automorphism groups of free groups, Thompson's groups, and even some elementary amenable groups.
\end{abstract}

Mathematics Subject Classification (2010). 20F65, 57M07, 20E06, 20E34, $20 \mathrm{E} 36$.

Keywords. Asymptotic properties of groups, groups presentable by products.

\section{Introduction}

In our previous paper [28] we introduced a class of infinite groups, called groups not presentable by products. Our motivation was that certain closed manifolds whose fundamental groups belong to this class turned out to have special properties; in particular some such manifolds are not dominated by non-trivial product manifolds. The purpose of the present paper is to discuss groups not presentable by products more systematically, and, in particular, to provide further examples of such groups, going far beyond the examples given in [28]. First, we recall the definition.

Definition 1.1 ([28]). An infinite group $\Gamma$ is not presentable by a product if, for every homomorphism $\varphi: \Gamma_{1} \times \Gamma_{2} \rightarrow \Gamma$ onto a subgroup of finite index, one of the factors $\Gamma_{i}$ has finite image $\varphi\left(\Gamma_{i}\right) \subset \Gamma$.

For the fundamental groups of closed Riemannian manifolds of strictly negative sectional curvature this property holds, essentially by the proof of Preissmann's theorem. Generalizing this observation, we previously proved:

\footnotetext{
*The first author is grateful to the Institute for Advanced Study in Princeton, respectively the Mathematical Sciences Research Institute in Berkeley, for hospitality at the beginning, respectively at the end, of the work on this paper. The second author is grateful for the support of the Hausdorff Research Institute for Mathematics in Bonn during the HIM Trimester Program "Rigidity."
} 
Theorem 1.2 ([28], Theorem 1.5). The following groups are not presentable by products:

(H) hyperbolic groups that are not virtually cyclic,

(N-P) fundamental groups of closed Riemannian manifolds of non-positive sectional curvature of rank one and of dimension $\geq 2$,

(MCG) mapping class groups of closed oriented surfaces of genus $\geq 1$.

As a consequence of the discussion in this paper, we extend Theorem 1.2 in several directions.

After the preliminary Section 2, this paper consists of two main parts. In the first part, comprising Sections 3-6, we develop criteria to show that groups are not presentable by products. Although numerous examples are interspersed in this first part, we then devote the second part of the paper, comprising Sections 7, 8 and 9, to systematically testing the criteria from the first part on certain interesting classes of groups, leading to further examples.

In Section 3 we discuss groups with acentral subgroups, a notion tailored to the analysis of centralisers à la Preissmann. This discussion subsumes most of the ad hoc arguments that went into the proof of Theorem 1.2, but it also applies to other interesting examples, such as free products and certain elementary amenable groups obtained as semidirect products.

In Section 4 we develop obstructions to presentability by products coming from $L^{2}$-Betti numbers, from cost in the sense of Levitt and Gaboriau, and from the rank gradient in the sense of Lackenby.

In Section 5 we consider the Powers property introduced by de la Harpe, and in Section 6 the second bounded cohomology with coefficients in the regular representation, as pioneered by Burger, Monod and Shalom. These considerations show that the hyperbolicity in statement $(\mathrm{H})$ of Theorem 1.2 can be replaced by a "cohomological negative curvature" assumption. Needless to say, these "negative curvature" obstructions do not apply to amenable groups, although some of them are not presentable by products and are amenable (sic!) to a direct analysis of centralisers à la Preissmann.

In Section 7 we test our criteria on Richard Thompson's groups, which are not elementary amenable, but could still be amenable. In Section 8 we discuss the automorphism groups of free groups, proving the natural generalization to this class of the statement about mapping class groups in Theorem 1.2. Finally in Section 9 we discuss groups with infinitely many ends, in particular free products and their applications to connected sum decompositions of manifolds dominated by products.

The final Section 10 contains some further extensions of our results, and a discussion of the context in geometric and measurable group theory. The Appendix summarizes the applicability of different criteria to various classes of groups.

Acknowledgement. This paper was begun in 2008 as we were trying to understand some comments that N. Monod kindly made on [28]. More recently, he has again offered some crucial insights. We are very grateful to N. Monod for his generous 
contributions to this work. In particular, most of the results and proofs in Sections 4.1, 4.2, 6 and 10.1 were suggested by him.

\section{Preliminaries}

Throughout this paper we only consider finitely generated groups. This restriction is necessary for some of the results we use, but represents no significant loss of generality. In particular, it is always satisfied when considering fundamental groups of compact manifolds, as in the context of the domination relation for manifolds [28].

We recall a few of the elementary properties of groups not presentable by products developed in [28]. We do not give any proofs in this section as all the results that need proof were proved in Section 3.1 of loc. cit.

Consider a homomorphism $\varphi: \Gamma_{1} \times \Gamma_{2} \rightarrow \Gamma$ of groups. Without loss of generality we can replace each $\Gamma_{i}$ by its image in $\Gamma$ under the restriction of $\varphi$, so that we may assume the factors $\Gamma_{i}$ to be subgroups of $\Gamma$ and $\varphi$ to be multiplication in $\Gamma$.

Lemma 2.1. If a group is not presentable by a product, then every finite index subgroup has finite centre.

The following is a kind of converse to this observation:

Proposition 2.2. If every subgroup of finite index in a group $\Gamma$ has trivial centre, then $\Gamma$ is irreducible if and only if it is not presentable by a product.

The proof of this proposition is based on the following lemma.

Lemma 2.3. Let $\Gamma_{1}, \Gamma_{2} \subset \Gamma$ be commuting subgroups of a group $\Gamma$ with the property that $\Gamma_{1} \cup \Gamma_{2}$ generates $\Gamma$. Then the multiplication homomorphism $\varphi: \Gamma_{1} \times \Gamma_{2} \rightarrow \Gamma$ is well defined and surjective and the following statements hold:

(1) the intersection $\Gamma_{1} \cap \Gamma_{2} \subset \Gamma$ is a subgroup of the centre of $\Gamma$, and

(2) the kernel of $\varphi$ is isomorphic to the Abelian group $\Gamma_{1} \cap \Gamma_{2}$.

This gives the following exact sequences:

$$
\begin{gathered}
1 \rightarrow \Gamma_{1} \cap \Gamma_{2} \rightarrow \Gamma \rightarrow \Gamma /\left(\Gamma_{1} \cap \Gamma_{2}\right) \rightarrow 1, \\
1 \rightarrow \Gamma_{1} \cap \Gamma_{2} \rightarrow \Gamma_{1} \times \Gamma_{2} \rightarrow \Gamma \rightarrow 1 .
\end{gathered}
$$

Sometimes it is convenient to replace a given group by a subgroup of finite index. This transition does not affect presentability by products by the following straightforward observation:

Lemma 2.4. Let $\Gamma$ be a group. A finite index subgroup of $\Gamma$ is presentable by a product if and only if $\Gamma$ is. 


\section{Acentral subgroups and acentral extensions}

In this section we define groups with acentral subgroups and acentral extensions and prove that they are not presentable by products. We shall give various examples, including in particular elementary amenable groups that are not presentable by products because they are extensions of this type.

Definition 3.1. Let $\Gamma$ be a group. A subgroup $A$ of $\Gamma$ is called acentral if for every $g \in A \backslash\{1\} \subset \Gamma$ the centraliser $C_{\Gamma}(g)$ is contained in $A$.

An extension $1 \rightarrow N \rightarrow \Gamma \rightarrow Q \rightarrow 1$ of groups is acentral if the normal subgroup $N$ is acentral.

Our interest in these notions stems from the following result.

Proposition 3.2. Groups containing infinite acentral subgroups of infinite index are not presentable by products.

Proof. The proof, in the same spirit as the ad hoc arguments of [28], consists of a careful analysis of the commutation relations in a group $\Gamma$ containing an infinite acentral subgroup $A$ of infinite index.

Assume for a contradiction that $\Gamma$ is presentable by a product. Then there are commuting infinite subgroups $\Gamma_{1}$ and $\Gamma_{2}$ such that the multiplication homomorphism $\varphi: \Gamma_{1} \times \Gamma_{2} \rightarrow \Gamma$ is well defined and has finite index image in $\Gamma$.

As a first step we show that $\Gamma_{i} \cap A=1$. If $\Gamma_{1} \cap A$ contained a non-trivial element $g$, then-because $A$ is acentral and the $\Gamma_{i}$ commute - we would obtain $\Gamma_{2} \subset C_{\Gamma}(g) \subset A$. Applying acentrality again, we then deduce that also $\Gamma_{1} \subset A$. But then $\operatorname{im} \varphi \subset A$, contradicting the fact that $\operatorname{im} \varphi$ has finite index in $\Gamma$ and $A$ has infinite index in $\Gamma$. Therefore, indeed $\Gamma_{1} \cap A=1$ and $\Gamma_{2} \cap A=1$.

As a second step we show that even $(\operatorname{im} \varphi) \cap A=1$. Assume for a contradiction that $(\operatorname{im} \varphi) \cap A \neq 1$ and let $g \in(\operatorname{im} \varphi) \cap A \backslash\{1\}$. Because $\Gamma_{1}$ and $\Gamma_{2}$ commute and because $\Gamma_{1} \cup \Gamma_{2}$ generates $\operatorname{im} \varphi$, we find elements $g_{1} \in \Gamma_{1}$ and $g_{2} \in \Gamma_{2}$ such that $g=g_{1} \cdot g_{2}$; notice that $g_{1} \in C_{\Gamma}(g)$. Therefore, acentrality implies that $g_{1} \in A$, and hence - by the first step - we have $g_{1}=1$. Applying the first step to $\Gamma_{2}$, we obtain also $g_{2}=1$, which contradicts $g \neq 1$. So $(\operatorname{im} \varphi) \cap A=1$.

As the third and last step we show that any subgroup $\Gamma^{\prime}$ of $\Gamma$ with $\Gamma^{\prime} \cap A=1$ cannot have finite index in $\Gamma$. Since $A$ is infinite, if $\Gamma^{\prime}$ had only finitely many cosets in $\Gamma$, then by the pigeonhole principle there would be a coset, say $g \Gamma^{\prime}$, containing infinitely many elements of $A$. In particular $g \Gamma^{\prime} \cap A$ would contain two elements $a_{1} \neq a_{2}$. But then it would follow that $1 \neq a_{2}^{-1} \cdot a_{1} \in \Gamma^{\prime} \cap A$, which would be a contradiction.

Combining the second and third steps we reach the conclusion that $\operatorname{im} \varphi$ cannot have finite index in $\Gamma$. Thus, $\Gamma$ is not presentable by a product after all. 
Corollary 3.3. Let $1 \rightarrow N \rightarrow \Gamma \rightarrow Q \rightarrow 1$ be an acentral extension of groups with $N$ and $Q$ infinite. Then $\Gamma$ is not presentable by a product.

Before proceeding, we would like to point out that many instances of the ad hoc arguments of [28] can be subsumed under the result of Proposition 3.2. For example, in the mapping class groups of surfaces of genus $\geq 3$ there are pseudo-Anosov elements $g$ with the property that the cyclic subgroup generated by $g$ is acentral. Thus the case (MCG) in Theorem 1.2 follows from Proposition 3.2. Note that it is crucial here that we consider acentral subgroups that are not necessarily normal. This is also true in the next example.

Example 3.4. Let $\Gamma=\Delta_{1} * \Delta_{2}$ be a non-trivial free product, and $g \in \Gamma$ an element that is not contained in a conjugate of one of the free factors, e.g., $g=g_{1} g_{2} g_{1}^{-1} g_{2}^{-1}$ with $g_{i} \in \Delta_{i} \backslash\{e\}$. Then the centraliser $C_{\Gamma}(g)$ is an infinite acentral subgroup of $\Gamma$; see [32], Problem 28 on p. 196. As soon as one of the $\Delta_{i}$ has order $>2$, the index of $C_{\Gamma}(g)$ in $\Gamma$ is also infinite, so that $\Gamma$ is not presentable by a product.

Corollary 3.5. Let $\Gamma$ denote the semi-direct product group $N \rtimes_{\alpha} Q$, where $N$ is anontrivial Abelian group, $Q$ is an infinite group and the action on $N$ given by $\alpha: Q \rightarrow$ $\operatorname{Aut}(N)$ is free outside $0 \in N$. Then the extension $0 \rightarrow N \rightarrow \Gamma \rightarrow Q \rightarrow 1$ is acentral and $N$ is infinite. In particular, $\Gamma$ is not presentable by a product.

Proof. In view of Corollary 3.3, it suffices to prove that $N$ is infinite and acentral. Infiniteness is clear since the infinite group $Q$ acts freely on the (non-empty) set $N \backslash\{0\}$.

Let $g \in N \backslash\{0\}$ and let $g^{\prime} \in C_{\Gamma}(g)$; we write $g=(n, 1)$ and $g^{\prime}=\left(n^{\prime}, q^{\prime}\right)$ with $n, n^{\prime} \in N$ and $q^{\prime} \in Q$. By definition of the semi-direct product, we obtain

$$
\begin{aligned}
& g \cdot g^{\prime}=\left(n+n^{\prime}, q^{\prime}\right), \\
& g^{\prime} \cdot g=\left(n^{\prime}+\alpha\left(q^{\prime}\right)(n), q^{\prime}\right),
\end{aligned}
$$

and hence $n+n^{\prime}=n^{\prime}+\alpha\left(q^{\prime}\right)(n)$; because $N$ is Abelian, we use " + " for the group structure in $N$. In particular, $n=\alpha\left(q^{\prime}\right)(n)$. As $\alpha$ acts freely on $N$ outside 0 and $n \neq 0$, this implies $q^{\prime}=1$, i.e., $g^{\prime} \in N \rtimes_{\alpha} 1=N$. Thus $N$ is indeed acentral.

This corollary provides us with explicit examples of elementary amenable groups that are not presentable by products, by taking semi-direct products of infinite amenable groups $Q$ with suitable actions on Abelian groups $N$. Note that the obstructions to presentability by products developed below coming from rank gradient, cost and $L^{2}$-Betti numbers (Section 4), from the Powers property (Section 5), or from bounded cohomology (Section 6), vanish for amenable groups.

Example 3.6. Let $\alpha: \mathbb{Z} \rightarrow \operatorname{SL}(2, \mathbb{Z})$ be given by the matrix

$$
A=\left(\begin{array}{ll}
2 & 1 \\
1 & 1
\end{array}\right) \text {. }
$$


Then the action of $\mathbb{Z}$ on $\mathbb{Z}^{2}$ given by $A$ is free outside 0 and Corollary 3.5 shows that the corresponding semi-direct product $\Gamma=\mathbb{Z}^{2} \rtimes_{\alpha} \mathbb{Z}$ is not presentable by a product. On the other hand, $\Gamma$ is solvable and thus amenable.

In this example $\Gamma$ is the fundamental group of a $T^{2}$-bundle $M$ over $S^{1}$ whose monodromy is the Anosov diffeomorphism given by $A$ acting linearly on $\mathbb{R}^{2} / \mathbb{Z}^{2}$. This torus bundle carries the solvable Thurston geometry $\mathrm{Sol}^{3}$, and $\Gamma$ is a lattice in $\mathrm{Sol}^{3}$. For all such lattices we have:

Corollary 3.7. Let $\Gamma$ be any cocompact lattice in $\mathrm{Sol}^{3}$. Then $\Gamma$ is not presentable by a product.

Proof. Any such lattice has a finite index subgroup that is the fundamental group of the mapping torus of a hyperbolic torus diffeomorphism; see [42], Theorem 5.3 (i). The discussion in Example 3.6 applies to this finite index subgroup.

The previous example can be generalized to higher dimensions.

Example 3.8. Let $n \in \mathbb{N}_{\geq 2}$ and let $\alpha: \mathbb{Z} \rightarrow \mathrm{GL}(n, \mathbb{Z})$ be given by a matrix $A \in$ $\mathrm{GL}(n, \mathbb{Z})$ such that no non-trivial power of $A$ has eigenvalue 1 . Then the action of $\mathbb{Z}$ on $\mathbb{Z}^{n}$ given by $A$ is free outside 0 and Corollary 3.5 shows that the corresponding semi-direct product $\mathbb{Z}^{n} \rtimes_{\alpha} \mathbb{Z}$ is not presentable by a product. Again the group is solvable and thus amenable.

\section{4. $L^{2}$-Betti numbers, cost, and rank gradient}

In this section we discuss obstructions to presentability by products coming from $L^{2}$-Betti numbers, from cost, and from the rank gradient.

4.1. $L^{2}$-Betti numbers. Like the ordinary Betti numbers, $L^{2}$-Betti numbers of groups can be defined as dimensions of certain homology modules, namely as the von Neumann dimensions of homology with coefficients in the group von Neumann algebra. For a thorough treatment of $L^{2}$-invariants we refer the reader to Lück's book [30].

The first $L^{2}$-Betti number is an obstruction for groups to be presentable by products:

Proposition 4.1. If the group $\Gamma$ is presentable by a product then $b_{1}^{(2)}(\Gamma)=0$.

Proof. Assuming that $\Gamma$ is presentable by a product we find two infinite commuting subgroups $\Gamma_{1}$ and $\Gamma_{2}$ of $\Gamma$ with the property that the multiplication homomorphism $\varphi: \Gamma_{1} \times \Gamma_{2} \rightarrow \Gamma$ is surjective onto a finite index subgroup $\Gamma^{\prime}:=\operatorname{im} \varphi$ in $\Gamma$. 
As $L^{2}$-Betti numbers are multiplicative with respect to finite index subgroups (see Theorem 6.54 (6) in [30]), we have

$$
b_{1}^{(2)}(\Gamma)=\frac{1}{\left[\Gamma: \Gamma^{\prime}\right]} \cdot b_{1}^{(2)}\left(\Gamma^{\prime}\right) .
$$

In particular, it suffices to prove that $b_{1}^{(2)}\left(\Gamma^{\prime}\right)=0$.

We now divide the discussion into two cases:

(1) The group $\Gamma_{1} \cap \Gamma_{2}$ is infinite. Since $\Gamma_{1} \cap \Gamma_{2}$ is Abelian, it is amenable. Thus, by the exact sequence (1), the group $\Gamma^{\prime}$ has an infinite amenable normal subgroup, which implies that $b_{1}^{(2)}\left(\Gamma^{\prime}\right)=0$; see [30], Theorem 7.2 (1) and (2).

(2) The group $\Gamma_{1} \cap \Gamma_{2}$ is finite. In this case the exact sequence (2) implies that

$$
b_{1}^{(2)}\left(\Gamma^{\prime}\right)=\left|\Gamma_{1} \cap \Gamma_{2}\right| \cdot b_{1}^{(2)}\left(\Gamma_{1} \times \Gamma_{2}\right)
$$

compare [30], Exercise 7.7 and p. 534 f. Moreover, $b_{1}^{(2)}\left(\Gamma_{1} \times \Gamma_{2}\right)$ can be computed by the Künneth formula [30], Theorem 6.54 (5), (8),

$$
\begin{aligned}
b_{1}^{(2)}\left(\Gamma_{1} \times \Gamma_{2}\right) & =b_{0}^{(2)}\left(\Gamma_{1}\right) \cdot b_{1}^{(2)}\left(\Gamma_{2}\right)+b_{1}^{(2)}\left(\Gamma_{1}\right) \cdot b_{0}^{(2)}\left(\Gamma_{2}\right) \\
& =\frac{1}{\left|\Gamma_{1}\right|} \cdot b_{1}^{(2)}\left(\Gamma_{2}\right)+b_{1}^{(2)}\left(\Gamma_{1}\right) \cdot \frac{1}{\left|\Gamma_{2}\right|} \\
& =0
\end{aligned}
$$

where the last equality holds because the groups $\Gamma_{1}$ and $\Gamma_{2}$ are infinite. Thus, it follows that $b_{1}^{(2)}\left(\Gamma^{\prime}\right)=0$.

Hence, we obtain $b_{1}^{(2)}(\Gamma)=1 /\left[\Gamma: \Gamma^{\prime}\right] \cdot b_{1}^{(2)}\left(\Gamma^{\prime}\right)=0$, as desired.

Remark 4.2. The vanishing result in Proposition 4.1 does not extend to the higher $L^{2}$-Betti numbers. However, the proof of case (1) does extend. Therefore, we do get restrictions on the higher $L^{2}$-Betti numbers of groups that are presentable by products. For example, if $\Gamma$ is presentable by a product and $b_{2}^{(2)}(\Gamma) \neq 0$, then $\Gamma$ is a quotient of a direct product $\Gamma_{1} \times \Gamma_{2}$ by a finite central subgroup, and both $b_{1}^{(2)}\left(\Gamma_{1}\right) \neq 0$ and $b_{1}^{(2)}\left(\Gamma_{2}\right) \neq 0$. This follows from the Künneth formula as in case (2) of the proof above.

4.2. Expensive groups. The concept of cost was introduced by Levitt and developed extensively by Gaboriau [15]. It is a dynamical/ergodic invariant of discrete groups. We shall use the lecture notes of Kechris and Miller [25] as our reference for this concept and for the properties we need.

The cost $\mathcal{C}(\Gamma)$ of a countable group $\Gamma$ is either infinite or a non-negative real number. For finite groups one has $\ell(\Gamma)=1-1 /|\Gamma|$, and for infinite groups one has $\varphi(\Gamma) \geq 1$. 
Definition 4.3 ([25]). An infinite countable group $\Gamma$ is cheap if $\mathcal{C}(\Gamma)=1$; it is expensive if $\mathcal{C}(\Gamma)>1$.

If $\Gamma^{\prime} \subset \Gamma$ is a subgroup of finite index, then $\mathcal{C}\left(\Gamma^{\prime}\right)-1=\left[\Gamma: \Gamma^{\prime}\right] \cdot(\mathcal{C}(\Gamma)-1)$ [15], Théorème VI.1, [25], Theorem 34.1. Therefore, the property of being cheap, or expensive, is invariant under passage to finite index sub- or supergroups. Similarly, if $\Gamma^{\prime}$ is a finite normal subgroup of $\Gamma$, then $\Gamma / \Gamma^{\prime}$ is cheap if and only if $\Gamma$ is cheap [15], Théorème VI.19.

Proposition 4.4. Expensive groups are not presentable by products.

Proof. Suppose that $\Gamma$ is expensive and presentable by a product. Then, as the property of being expensive is invariant under passage to finite index subgroups, Lemma 2.4 allows us to assume that we have commuting subgroups $\Gamma_{1}, \Gamma_{2} \subset \Gamma$ such that the multiplication $\varphi: \Gamma_{1} \times \Gamma_{2} \rightarrow \Gamma$ is surjective. If $\Gamma_{1} \cap \Gamma_{2}$ is infinite, then $\Gamma$ has infinite centre, and so $\Gamma$ is cheap [15], VI.26 (a), [25], Corollary 35.3. If $\Gamma_{1} \cap \Gamma_{2}$ is finite, then in view of the exact sequence (2) and the fact that the property of being cheap is invariant under passage to quotients by finite normal subgroups, it suffices to check that $\Gamma_{1} \times \Gamma_{2}$ is cheap. This last assertion is known to be true as soon as both $\Gamma_{i}$ are infinite [15], Proposition VI.23, [25], Theorem 33.3.

4.3. Rank gradient. The rank gradient was introduced by Lackenby [29], and has recently been further developed by Abért and Nikolov [1].

For any finitely generated group $\Gamma$, let $d(\Gamma)$ be the minimal number of generators. Then the rank gradient is defined to be

$$
\operatorname{RG}(\Gamma)=\inf _{\Gamma^{\prime} \subset \Gamma} \frac{d\left(\Gamma^{\prime}\right)-1}{\left[\Gamma: \Gamma^{\prime}\right]},
$$

with the infimum taken over all finite index subgroups $\Gamma^{\prime} \subset \Gamma$. (This is sometimes called the absolute rank gradient. Often only normal subgroups are considered, but this makes no difference.) Of course, if $\Gamma$ has few subgroups of finite index, this definition may not be very meaningful. In the extreme case when $\Gamma$ has no subgroups of finite index at all, one clearly has $\operatorname{RG}(\Gamma)=d(\Gamma)-1$. This explains why results about the rank gradient often involve assumptions that ensure the existence of sufficiently many finite index subgroups.

The basic properties of the rank gradient immediately give the following:

Proposition 4.5. If a residually finite group $\Gamma$ is presentable by a product, then $\operatorname{RG}(\Gamma)=0$.

Proof. Suppose $\Gamma$ is presentable by a product. Then there are infinite commuting subgroups $\Gamma_{1}, \Gamma_{2} \subset \Gamma$ such that the multiplication map $\varphi: \Gamma_{1} \times \Gamma_{2} \rightarrow \Gamma$ is surjective onto a finite index subgroup $\Gamma^{\prime} \subset \Gamma$. It suffices to prove $\operatorname{RG}\left(\Gamma^{\prime}\right)=0$. 
If $\Gamma_{1} \cap \Gamma_{2}$ is infinite, then $\Gamma^{\prime}$ has infinite centre, and so its rank gradient vanishes [1], Theorem 3.

If $\Gamma_{1} \cap \Gamma_{2}$ is finite, we argue as follows. By assumption, both $\Gamma_{i}$ are infinite. As they are subgroups of a residually finite group, they are themselves residually finite. As the two groups commute, they are both normal in $\Gamma^{\prime}$, and we have the two exact sequences

$$
\begin{aligned}
& 1 \rightarrow \Gamma_{1} \rightarrow \Gamma^{\prime} \rightarrow \Gamma_{2} /\left(\Gamma_{1} \cap \Gamma_{2}\right) \rightarrow 1, \\
& 1 \rightarrow \Gamma_{2} \rightarrow \Gamma^{\prime} \rightarrow \Gamma_{1} /\left(\Gamma_{1} \cap \Gamma_{2}\right) \rightarrow 1 ;
\end{aligned}
$$

the epimorphisms are given by composing the isomorphism $\Gamma^{\prime} \cong \Gamma_{1} \times \Gamma_{2} /\left(\Gamma_{1} \cap \Gamma_{2}\right)$ with the homomorphisms induced by the projection from $\Gamma_{1} \times \Gamma_{2}$ onto its factors. The lower sequence shows that $\Gamma_{1} /\left(\Gamma_{1} \cap \Gamma_{2}\right)$ is finitely generated, and since $\Gamma_{1} \cap \Gamma_{2}$ is finite, we conclude that $\Gamma_{1}$ is itself finitely generated (recall that $\Gamma$ (and hence $\Gamma^{\prime}$ ) is finitely generated by our standing convention). Now we can apply a result of Abért and Nikolov (Proposition 13 in [1]) to the first extension above to conclude the vanishing of $\operatorname{RG}\left(\Gamma^{\prime}\right)$. The subgroup $\Gamma_{1}$ is finitely generated, and the quotient $\Gamma_{2} /\left(\Gamma_{1} \cap \Gamma_{2}\right)$ has subgroups of arbitrarily large index since $\Gamma_{2}$ is infinite and residually finite, and $\Gamma_{1} \cap \Gamma_{2}$ is finite. This completes the proof.

Example 4.6. Let $\Gamma$ be a finitely generated infinite simple group. By classical work of Higman and Thompson, such groups exist, and may even be chosen to be finitely presentable. Then $\Gamma \times \Gamma$ is presentable by a product and has positive rank gradient since it has no proper subgroups of finite index.

Note that $\Gamma$ itself is not presentable by a product since it has no non-trivial normal subgroups.

\subsection{The relation between the first $L^{2}$-Betti number, cost, and the rank gradient.} There is a remarkable connection between cost and the first $L^{2}$-Betti number, which shows that the obstruction to presentability by a product coming from the first $L^{2}$-Betti number is a special case of the obstruction provided by the cost.

Theorem 4.7 (Gaboriau [16], Corollaire 3.23). Every infinite group $\Gamma$ satisfies $\digamma(\Gamma)-1 \geq b_{1}^{(2)}(\Gamma)$. In particular, groups with positive first $L^{2}$-Betti numbers are expensive.

It is unknown whether this inequality is ever strict. For residually finite groups one also has:

Theorem 4.8 (Abért and Nikolov [1], Theorem 1). If $\Gamma$ is residually finite, then $\mathrm{RG}(\Gamma) \geq €(\Gamma)-1$, with equality if $\Gamma$ has fixed price.

We refer the reader to the papers by Abért and Nikolov [1] and by Osin [40] for further discussions of these results and their relations to open problems and conjectures in group theory and in three-dimensional topology. 
For residually finite groups, the positivity of the rank gradient is the strongest one of the three obstructions to presentability by products discussed in this section. A large class of non-examples for this obstruction comes from the following observation, generalising a vanishing result for $L^{2}$-Betti numbers [30], Theorem 1.39.

Lemma 4.9 (Lackenby [29], p. 365-366). The rank gradient vanishes for fundamental groups of mapping tori.

Example 4.10. By Thurston's theorem, the mapping torus of a pseudo-Anosov diffeomorphism of a surface of genus $\geq 2$ is a hyperbolic three-manifold. Its fundamental group is residually finite, therefore the vanishing of its rank gradient given by Lemma 4.9 implies that this hyperbolic group is cheap.

Another non-example is the following:

Proposition 4.11. Mapping class groups of surfaces of genus $\geq 3$ have vanishing rank gradient.

Since mapping class groups are residually finite by a result of Grossman [18], this Proposition implies, via Theorem 4.8, that mapping class groups are cheap. This last assertion was previously known by a recent result due to Kida [26].

Proof. Let $\Gamma_{g}$ be the mapping class group of a closed oriented surface of genus $g \geq 3$. We can apply a result of Abért and Nikolov (Proposition 13 of [1]) to the extension

$$
1 \rightarrow \mathcal{I}_{g} \rightarrow \Gamma_{g} \rightarrow \operatorname{Sp}(2 g ; \mathbb{Z}) \rightarrow 1
$$

to conclude $\operatorname{RG}\left(\Gamma_{g}\right)=0$. Here $\mathcal{I}_{g}$ is the Torelli group, which is finitely generated for genus $\geq 3$ by a result of Johnson [24]. Clearly the symplectic group has the required property to admit finite quotients of arbitrarily large order.

\section{The Powers property}

For a countable group $\Gamma$, let $C_{\text {red }}^{*}(\Gamma)$ denote its reduced $C^{*}$-algebra. One says that the group is $C^{*}$-simple if $C_{\text {red }}^{*}(\Gamma)$ has no proper two-sided ideals. It is a now classical result of Powers that the free group on two generators is $C^{*}$-simple. De la Harpe [22] extracted from Powers's proof a combinatorial property of groups that ensures $C^{*}$-simplicity. He calls this property the Powers property, and calls groups that have the property Powers groups. We refer to his recent survey [23] for the definitions and an extensive bibliography of results on the class $\mathcal{P}$ of Powers groups.

Basic results about Powers groups mentioned in [23] imply the following.

Proposition 5.1. A group with the Powers property is not presentable by products. 
Proof. First of all, if $\Gamma$ is a Powers group, so is every finite index subgroup [22], Proposition 1 (c). Therefore, if we have subgroups $\Gamma_{1}, \Gamma_{2} \subset \Gamma$ for which the multiplication is surjective onto a finite index subgroup, we just pass to this subgroup. Now a $C^{*}$-simple group does not contain any amenable normal subgroup, in particular it has trivial centre. Therefore, by the discussion in Section 2, we conclude that $\Gamma_{1} \times \Gamma_{2}$ is a Powers group. But this contradicts a result of Promislow [41]; compare also [23], Proposition 14 (i).

This result is true for Powers groups only. The less restrictive property of $C^{*}$ simplicity is preserved under taking direct products, and so cannot be an obstruction against presentability by products. The same remark applies to the "weak Powers property" discussed in [23].

The fact that $C^{*}$-simple groups, and therefore Powers groups, have trivial centre, implies that many standard examples cannot be Powers groups.

Example 5.2. The centre of $\operatorname{SL}(2, \mathbb{Z})$ has order 2. Therefore, this is not a Powers group. It follows that hyperbolic groups, or groups with infinitely many ends, are not always Powers groups.

Example 5.3. The mapping class group of a closed genus 2 surface also has centre of order 2, generated by the hyperelliptic involution. It follows that this mapping class group is not a Powers groups. Similarly, in higher genus the hyperelliptic mapping class group is not a Powers group.

Nevertheless, the class $\mathcal{P}$ of Powers groups contains, among others, the following groups:

(1) torsion-free hyperbolic groups that are not virtually cyclic (de la Harpe [22], [23]),

(2) free products $\Delta_{1} * \Delta_{2}$ with $\left|\Delta_{i}\right|>i$ (de la Harpe [22], Proposition 8),

(3) mapping class groups of surfaces of genus at least 3 (Bridson-de la Harpe [10], Theorem 2.2).

\section{Bounded cohomology}

Monod and Shalom [37], [38] introduced and studied the following class of groups; compare also the paper by Mineyev, Monod and Shalom [34]. (A detailed treatment of bounded cohomology $H_{b}^{*}$ of groups is given in Monod's book [35]).

Definition 6.1 ([38]). A countable group $\Gamma$ is in $\mathcal{C}_{\text {reg }}$ if $H_{b}^{2}\left(\Gamma ; \ell^{2}(\Gamma)\right) \neq 0$.

The class $\mathcal{C}_{\text {reg }}$ contains, among others, the following groups: 
(1) hyperbolic groups that are not virtually cyclic (Mineyev-Monod-Shalom [34], Theorem 3; see also [37], [19]),

(2) groups with infinitely many ends (Monod-Shalom [37], Corollary 7.9),

(3) mapping class groups of surfaces of genus at least 2 (Hamenstädt [19], Theorem 4.5).

The results of Hamenstädt [19] hold more generally for all groups acting by isometries on a Gromov-hyperbolic space, as long as the action satisfies a so-called weak acylindricity property.

Proposition 6.2. Groups in the class $\mathcal{C}_{\text {reg }}$ are not presentable by products.

Proof. This is implicit in the work of Monod and Shalom [38], Section 7.

Assume for a contradiction that $\Gamma$ is a group in $\ell_{\text {reg }}$ that is presentable by a product. If a group is in $\ell_{\text {reg }}$, then so are all its finite index subgroups $\Gamma$ [38], Lemma 7.5. Therefore, by Lemma 2.4, we may assume that $\Gamma$ contains commuting subgroups $\Gamma_{1}$ and $\Gamma_{2}$ such that the multiplication homomorphism $\Gamma_{1} \times \Gamma_{2} \rightarrow \Gamma$ is surjective. Now if $\Gamma_{1} \cap \Gamma_{2}$ is infinite, then $\Gamma$ contains an infinite amenable normal subgroup by the exact sequence (1), which contradicts the assumption that $\Gamma$ is in $\mathcal{C}_{\text {reg }}$ [38], Proposition 7.10 (ii). If $\Gamma_{1} \cap \Gamma_{2}$ is finite, then the exact sequence (2) and Lemma 7.3 in [38] imply that $\Gamma_{1} \times \Gamma_{2}$ is in $\ell_{\text {reg. }}$. If both $\Gamma_{i}$ are infinite, this is impossible [38], Proposition 7.10 (iii).

Proposition 6.2 can be generalized in two different directions. On the one hand, one can consider the class $\ell$ of groups for which $H_{b}^{2}(\Gamma ; \pi) \neq 0$ for some mixing unitary representation $\pi$ of $\Gamma$, which is not necessarily the regular representation $\ell^{2}(\Gamma)$. The class $\mathcal{C}$ was also introduced by Monod and Shalom [38], and their results used above for $\ell_{\text {reg }}$ apply more generally to $\ell$. It is at present unknown whether the inclusion $\ell_{\text {reg }} \subset \ell$ is strict. On the other hand, Thom [43] has introduced the following variant of $\varphi_{\text {reg }}$.

Definition 6.3 ([43]). A countable group $\Gamma$ is in $\mathscr{D}_{\text {reg }}$ if $\operatorname{dim}_{L \Gamma} Q H^{1}\left(\Gamma ; \ell^{2}(\Gamma)\right) \neq 0$, where $Q H^{1}$ denotes the first quasi-cohomology and $L \Gamma$ is the group von Neumann algebra of $\Gamma$.

It is as yet unknown whether $\boldsymbol{C}_{\text {reg }}$ and $\mathscr{D}_{\text {reg }}$ agree. As far as presentability by products goes, both are equally good:

Proposition 6.4. Groups in $\mathfrak{D}_{\text {reg }}$ are not presentable by products.

Proof. A standard exact sequence argument shows that if $\Gamma$ is in $\mathscr{D}_{\text {reg }}$ then $b_{1}^{(2)}(\Gamma) \neq$ 0 or $\Gamma$ is in $\ell_{\text {reg }}$ [43], Lemma 2.8. In the first case the conclusion follows from Proposition 4.1, in the second case it follows from Proposition 6.2. 


\section{Thompson's groups}

Richard Thompson's groups $F, T$ and $V$ are interesting test cases for many issues in group theory. We refer to the survey by Cannon et. al. [11] for their basic properties.

The groups $T$ and $V$ are simple, and are therefore trivially not presentable by products. For $F$ we have:

Proposition 7.1. The Thompson group $F$ is not presentable by products.

Proof. Suppose $\Gamma_{1}, \Gamma_{2} \subset F$ are commuting infinite subgroups such that the multiplication map $\Gamma_{1} \times \Gamma_{2} \rightarrow F$ is surjective onto a finite index subgroup $\Gamma \subset F$. There is a normal finite index subgroup $\bar{\Gamma} \subset F$ contained in $\Gamma$. Since $\bar{\Gamma}$ is normal in $F$, it contains the commutator subgroup $[F, F]$ by [11], Theorem 4.3. Since $[F, F]$ is normal in $F$, it is also normal in $\Gamma$. The quotient $\Gamma /[F, F]$ is Abelian.

Since $\Gamma_{i}$ and $[F, F]$ are both normal in $\Gamma$, their intersection $\bar{\Gamma}_{i}=\Gamma_{i} \cap[F, F]$ is normal in $[F, F]$. However, $[F, F]$ is a simple group [11], Theorem 4.5. Thus $\bar{\Gamma}_{i}$ is trivial or all of $[F, F]$. If $\bar{\Gamma}_{i}$ is trivial, then the composition

$$
\Gamma_{i} \hookrightarrow \Gamma \rightarrow \Gamma /[F, F]
$$

is injective, and so $\Gamma_{i}$ must be Abelian. But then $\Gamma_{i}$ is an infinite central subgroup of $\Gamma$. This contradicts the fact that every finite index subgroup of $F$ must have trivial centre. For $F$ itself this is proved in [11], p. 229, and that proof applies to all finite index subgroups.

The only possibility left is that both $\bar{\Gamma}_{i}$ equal $[F, F]$. But then $[F, F]$ is contained in $\Gamma_{1} \cap \Gamma_{2}$, and so must be Abelian by the discussion in Section 2 . This contradicts the fact that $[F, F]$ is an infinite simple group by [11], Theorem 4.5 . This contradiction proves that $F$ can not be presentable by a product.

This proposition can not be proved using the rank gradient, cost or the first $L^{2}$-Betti number, since $F$ contains copies of itself with positive index $>1$, which immediately implies the vanishing of its rank gradient, and the vanishing of $\mathcal{C}(F)-1$ and of $b_{1}^{(2)}(F)$. In spite of various recent claims, at the time of writing it seems to be still unknown whether $F$ is amenable. If this were true, it would imply that the bounded cohomology of $F$ is trivial, and that $F$ is not $C^{*}$-simple, in particular $F$ would not be a Powers group. Note however that $F$ is not elementary amenable [11], Theorem 4.10, and so this is certainly a very different example from the elementary amenable groups discussed in Example 3.6 and Corollary 3.7.

\section{Automorphism groups of free and free Abelian groups}

In this section we test the obstructions against presentability by products in the examples of automorphism groups of free Abelian as well as non-Abelian free groups. In both cases we prove that the groups in question are not presentable by products. 
8.1. Automorphism groups of free Abelian groups. The questions of presentability by products for $\operatorname{GL}(n, \mathbb{Z})$ and for $\operatorname{SL}(n, \mathbb{Z})$ are equivalent, since the latter is a finite index subgroup in the former. These groups are residually finite with vanishing rank gradient [29], as shown by the consideration of congruence subgroups. Thus the obstructions of Section 4 do not apply. Moreover, for $n \in \mathbb{N}_{\geq 3}$, the groups $\operatorname{GL}(n, \mathbb{Z})$ and $\operatorname{SL}(n, \mathbb{Z})$ are not in the class $\mathcal{C}_{\text {reg }}$ [37], Theorem 1.4. These groups are not $C^{*}$ simple, since they have non-trivial centres. However, it is known that $\operatorname{PSL}(n, \mathbb{Z})$ is $C^{*}$-simple by a result of Bekka, Cowling and de la Harpe [5]. Whether PSL $(n, \mathbb{Z})$ is a Powers group for $n \geq 3$ is unknown; compare [10], [23]. Thus none of the high-tech obstructions can be used to prove that for any $n \geq 2$ the groups $\operatorname{SL}(n, \mathbb{Z})$ are not presentable by products. Nevertheless, this is true, as it is a special case of the following:

Proposition 8.1. Suppose $G$ is a connected semisimple Lie group with finite centre and rank $\geq 2$. If $\Gamma \subset G$ is an irreducible lattice, then $\Gamma$ is not presentable by products.

Proof. Assume for a contradiction that $\Gamma_{1}, \Gamma_{2} \subset \Gamma$ are infinite commuting subgroups such that the multiplication map $\Gamma_{1} \times \Gamma_{2} \rightarrow \Gamma$ is surjective onto a finite index subgroup $\bar{\Gamma} \subset \Gamma$. Then $\bar{\Gamma}$ is also an irreducible lattice. Since the $\Gamma_{i}$ are infinite normal subgroups in $\bar{\Gamma}$, the Margulis normal subgroup theorem (see [33], Chapter IV, [44], Chapter 8) implies that they have finite index in $\bar{\Gamma}$. Thus their intersection, which is a central subgroup, also has finite index, and so $\Gamma$ is virtually Abelian. This is absurd.

Of course, for the case of $\operatorname{GL}(n, \mathbb{Z})$ there is also an elementary argument. One can find two elements in $\operatorname{GL}(n, \mathbb{Z})$ that are diagonalizable over $\mathbb{C}$ and (whose non-trivial powers) have no non-trivial common proper invariant subspace in $\mathbb{C}^{n}$. Hence, the elements of a finite index subgroup of $\mathrm{GL}(n, \mathbb{Z})$ can not have a common invariant subspace in $\mathbb{C}^{n}$. Assume that $\mathrm{GL}(n, \mathbb{Z})$ were presentable by a product of subgroups $\Gamma_{1}$ and $\Gamma_{2}$. Using the fact that $\Gamma_{1}$ and $\Gamma_{2}$ commute, one could find a non-zero subspace $E \subset \mathbb{C}^{n}$ on which all elements of one of the factors, say $\Gamma_{2}$, act as multiples of the identity, and such that this subspace would also be $\Gamma_{1}$-invariant. Thus $E$ would be $\left(\Gamma_{1} \cup \Gamma_{2}\right)$-invariant. It would follow by what we said at the beginning that $E=\mathbb{C}^{n}$, contradicting the assumption that $\Gamma_{2}$ is infinite.

8.2. Automorphism groups of non-Abelian free groups. Let $F_{n}$ be a free group on $n>1$ generators, $\operatorname{Aut}\left(F_{n}\right)$ its automorphism group, and $\operatorname{Out}\left(F_{n}\right)=\operatorname{Aut}\left(F_{n}\right) / \operatorname{Inn}\left(F_{n}\right)$ its group of outer automorphisms. We use the following terminology:

Definition 8.2. An element in $\operatorname{Out}\left(F_{n}\right)$ is called reducible if it leaves invariant the conjugacy class of a free factor in $F_{n}$, and it is called irreducible otherwise.

An element $g \in \operatorname{Out}\left(F_{n}\right)$ is called fully irreducible if $g^{k}$ is irreducible for all $k \neq 0$. 
Fully irreducible elements are sometimes called irreducible with irreducible powers (iwip), cf. [31]. In $\operatorname{Out}\left(F_{n}\right)$ these elements play a rôle analogous to that of pseudo-Anosov elements in mapping class groups.

We now prove:

Proposition 8.3. If $n \in \mathbb{N}_{>1}$, then the groups $\operatorname{Aut}\left(F_{n}\right)$ and $\operatorname{Out}\left(F_{n}\right)$ are not presentable by products.

Proof. We begin with the case of $\operatorname{Out}\left(F_{n}\right)$. For $n=2$ this reduces to GL(2, $\left.\mathbb{Z}\right)$, so there is nothing to prove. For $n \geq 3$ we may appeal to Proposition 5.1, $\operatorname{since} \operatorname{Out}\left(F_{n}\right)$ is a Powers group by a result of Bridson and de la Harpe [10], Theorem 2.6.

Instead of using the Powers property, we can give a direct proof by contradiction. It follows from a result of Baumslag and Taylor [4], Proposition 1, that $\operatorname{Out}\left(F_{n}\right)$ is virtually torsion-free. Thus, by the discussion in Section 2, we may assume that we have a torsion-free finite index subgroup $\Gamma \subset \operatorname{Out}\left(F_{n}\right)$ together with two non-trivial commuting subgroups $\Gamma_{1}, \Gamma_{2} \subset \Gamma$ such that the multiplication homomorphism $\Gamma_{1} \times$ $\Gamma_{2} \rightarrow \Gamma$ is surjective.

Since $\Gamma$ has finite index in $\operatorname{Out}\left(F_{n}\right)$, there exists a fully irreducible element $g \in \Gamma$. By a result of Lustig [31] the centraliser $C_{\Gamma}(g)$ of $g$ is virtually cyclic. Related statements appear in the work of Bestvina, Feighn and Handel on the Tits alternative for $\operatorname{Out}\left(F_{n}\right)$; see e.g. Theorem 2.14 of [8].

We can write $g=g_{1} \cdot g_{2}$ with certain $g_{1} \in \Gamma_{1}$ and $g_{2} \in \Gamma_{2}$. As $g$ is non-trivial, we may assume that so is $g_{1}$; note that $g_{1} \in C_{\Gamma}(g)$. Moreover, there exists an element $g_{2}^{\prime} \in \Gamma_{2} \backslash\{1\}$ with $g_{2}^{\prime} \in C_{\Gamma}(g)$. If $g_{2} \neq 1$ then we can take $g_{2}^{\prime}=g_{2}$, and if $g_{2}=1$ we may choose any non-trivial element of $\Gamma_{2}$ for $g_{2}^{\prime}$. As both $g_{1}$ and $g_{2}^{\prime}$ have infinite order and are contained in the virtually cyclic group $C_{\Gamma}(g)$, they have common non-trivial powers. This shows that $\Gamma_{1} \cap \Gamma_{2}$ is infinite, and so the centre of $\Gamma$ is infinite by Lemma 2.3 .

This is a contradiction, since $\Gamma$ must in fact have trivial centre; compare [8]. (One way to see this is to check that $\Gamma$ contains two fully irreducible elements with distinct stable and unstable laminations.) This completes the direct proof that $\operatorname{Out}\left(F_{n}\right)$ is not presentable by products.

Next consider the extension

$$
1 \rightarrow F_{n} \rightarrow \operatorname{Aut}\left(F_{n}\right) \stackrel{\pi}{\rightarrow} \operatorname{Out}\left(F_{n}\right) \rightarrow 1 .
$$

We may pull back this extension to a torsion-free finite index subgroup of $\operatorname{Out}\left(F_{n}\right)$, so that the assumption on the quotient in [28], Proposition 3.9, is satisfied by what we just proved. Now [28], Proposition 3.9, tells us that $\operatorname{Aut}\left(F_{n}\right)$ is not presentable by a product since the extension (3) does not split when restricted to any finite index subgroups. This completes the proof of Proposition 8.3.

Remark 8.4. The direct argument for $\operatorname{Out}\left(F_{n}\right)$ could be rephrased to argue that the infinite cyclic subgroups generated by certain fully irreducible elements are acentral. 
Remark 8.5. After we proved directly that $\operatorname{Out}\left(F_{n}\right)$ is not presentable by products, we tried to find out whether $\operatorname{Out}\left(F_{n}\right)$ is (known to be) in $\mathcal{C}_{\text {reg. }}$. In reply to our question, Bestvina and Fujiwara told us that a proof of this statement will be contained in a forthcoming paper [7]. Since then, Hamenstädt [21] has given such a proof.

The rank gradient, the cost, or the first $L^{2}$-Betti number cannot be used to prove Proposition 8.3 in view of our next result:

Proposition 8.6. Let $n \in \mathbb{N}_{\geq 3}$. The groups $\operatorname{Aut}\left(F_{n}\right)$ and $\operatorname{Out}\left(F_{n}\right)$ are cheap. Their first $L^{2}$-Betti numbers and their rank gradients vanish.

Proof. The groups in question are residually finite. For $\operatorname{Aut}\left(F_{n}\right)$ this is a classical result of Baumslag, whereas for $\operatorname{Out}\left(F_{n}\right)$ it was proved by Grossman [18]. Thus, by the discussion in Section 4.4, we only have to prove the vanishing of the rank gradient. For this we use again the result of Abért and Nikolov ([1], Proposition 13) about extensions. For $\operatorname{Aut}\left(F_{n}\right)$ we apply the result to the extension (3). The group on the left is finitely generated and the group on the right admits finite quotients of arbitrarily large order.

Similarly for $\operatorname{Out}\left(F_{n}\right)$ we consider the extension

$$
1 \rightarrow \mathrm{IA}_{n} \rightarrow \operatorname{Out}\left(F_{n}\right) \rightarrow \mathrm{GL}(n ; \mathbb{Z}) \rightarrow 1 .
$$

The groups on the left and on the right are infinite, and the kernel $\mathrm{IA}_{n}$ is finitely generated by a classical result of Magnus. Again the group on the right has finite quotients of arbitrarily large order.

Remark 8.7. The argument for $\operatorname{Aut}\left(F_{n}\right)$ also works for $n=2$. The argument for $\operatorname{Out}\left(F_{n}\right)$ however breaks down for $n=2$ since $\mathrm{IA}_{2}$ is trivial. In this case $\operatorname{Out}\left(F_{2}\right)=\mathrm{GL}(2 ; \mathbb{Z})$ has positive rank gradient as it is virtually free [29].

\section{Ends, free products, and connected sums}

In this section we consider free products of groups, and, more generally, groups with infinitely many ends.

Proposition 9.1. Groups with infinitely many ends are not presentable by products.

Proof. It is well known that groups with infinitely many ends have positive first $L^{2}$ Betti number; see for example [3], Chapter 4, or [6], Corollary 1. Therefore the result follows from Proposition 4.1.

Alternatively we could use the fact that groups with infinitely many ends are in $\boldsymbol{C}_{\text {reg }}$, as proved by Monod and Shalom [37], Corollary 7.9, and appeal to Proposition 6.2. Notice however, that in contrast to the result about the first $L^{2}$-Betti number, the proof 
of Monod and Shalom uses Stallings's structure theorem for groups with infinitely many ends.

Finally, a completely elementary argument is possible as well. Freudenthal and Hopf proved that a group with infinitely many ends cannot be a direct product of infinite groups. The argument given by Freudenthal [13], 7.10, in fact proves the more general statement of this theorem. For the convenience of the reader we repeat this argument briefly.

Let $\Gamma$ be a group with infinitely many ends, and $\Gamma_{1}, \Gamma_{2}$ commuting infinite subgroups for which the multiplication map $\varphi: \Gamma_{1} \times \Gamma_{2} \rightarrow \Gamma$ is surjective onto a finite index subgroup. Since the number of ends is unchanged by passage to a finite index subgroup, we may assume that $\varphi$ is surjective. The assumption that $\Gamma$ has more than one end implies that there is an element $g \in \Gamma$ of infinite order for which $g^{n}$ and $g^{-n}$ belong to two different ends $e$ and $e^{\prime}$ as $n \rightarrow \infty$; see [13], 7.6.

Under the action of $\Gamma$ on its space of ends, the infinite cyclic subgroup $T$ generated by $g$ fixes $e$ and $e^{\prime}$. Write $g=g_{1} g_{2}$ with $g_{i} \in \Gamma_{i}$. The $g_{i}$ commute with $T$, and so both $g_{i}$ also fix $e$ and $e^{\prime}$. Since $g$ has infinite order, we may assume that so does $g_{1}$. Then $g_{1}$ generates an infinite cyclic subgroup $T^{\prime}$ of $\Gamma_{1}$ that fixes $e$ and $e^{\prime}$. As $\Gamma_{2}$ commutes with $T^{\prime}$, it contains a subgroup $\Gamma_{2}^{\prime}$ of index at most 2 that also fixes e and $e^{\prime}$ [13], 7.7. As $\Gamma_{1}$ commutes with $\Gamma_{2}^{\prime}$, it contains a subgroup $\Gamma_{1}^{\prime}$ of index at most 2 which also fixes $e$ and $e^{\prime}$ [13], 7.7. Thus $\Gamma$ has a subgroup of index at most 4 which fixes $e$ and $e^{\prime}$. This contradicts the assumption that $\Gamma$ has infinitely many ends.

Corollary 9.2. Let $\Delta_{1}$ and $\Delta_{2}$ be two non-trivial groups. Then the free product $\Delta_{1} *$ $\Delta_{2}$ is presentable by a product if and only if $\Delta_{1} \cong \mathbb{Z} / 2 \cong \Delta_{2}$.

Proof. On the one hand, $\mathbb{Z} / 2 * \mathbb{Z} / 2$ is virtually infinite cyclic, and therefore presentable by a product. On the other hand, if one of the groups has order at least 3, then their free product has infinitely many ends whence Proposition 9.1 applies. Alternatively we can use Example 3.4 to see that there are infinite acentral subgroups of infinite index and apply Proposition 3.2, or we can use Proposition 5.1 in conjunction with the fact that these free products are Powers groups; see Bridson and de la Harpe [10], Theorem 2.2.

Remark 9.3. Lackenby [29], Proposition 3.2, proved that the rank gradient of a free product $\Delta_{1} * \Delta_{2}$ of non-trivial groups is positive if at least one of the free factors has order $>2$. Therefore, for residually finite groups Corollary 9.2 also follows from Proposition 4.5.

We can use the last corollary to put restrictions on the connected sum decompositions of manifolds dominated by products. Suppose $N=N_{1} \# N_{2}$ is a connected sum of two closed oriented $n$-manifolds, and $P=X_{1} \times X_{2}$ is a non-trivial product of closed oriented manifolds with $P \geq N$. Then, collapsing one or the other summand of $N$ to a point, we see that $P \geq N_{1}$ and $P \geq N_{2}$. Thus, for $N$ to be dominated 
by a product it is necessary that its connected summands $N_{i}$ are also dominated by products. However, this necessary condition is not sufficient.

Theorem 9.4. If $N$ is a closed, oriented, connected rationally essential manifold that is dominated by a non-trivial product $P \geq N$ and that admits a connected sum decomposition $N=N_{1} \# N_{2}$, then one of the summands is simply connected, and the fundamental group of the other summand is presentable by a product.

Proof. Clearly we may assume that $N$ has dimension $\geq 3$. Then its fundamental group is the free product of the fundamental groups of the $N_{i}$, and, since $N$ is assumed rationally essential, at least one of these free factors must be infinite. If the other free factor is non-trivial, Corollary 9.2 tells us that $\pi_{1}(N)$ is not presentable by a product, which contradicts [28], Theorem 1.4. Thus one of the $N_{i}$ is simply connected, the other one is rationally essential, and its fundamental group is presentable by a product by [28], Theorem 1.4.

Example 9.5. In every dimension $n \geq 2$, the $n$-torus $T^{n}$ is a product, but $T^{n} \# T^{n}$ is not dominated by a product.

Remark 9.6. Notice however, that not all non-trivial connected sums are not dominated by a product; for instance, $\mathbb{C} P^{2} \# \mathbb{C} P^{2}$ is dominated by a product [28], Proposition 7.1.

\section{Final remarks}

10.1. Extension to subnormal subgroups. In this paper we have proved that various groups are not presentable by products. By definition, this notion refers to all subgroups of finite index, in particular the finite index normal subgroups. It turns out that in many cases one can treat all infinite normal subgroups of our groups, regardless of whether they have finite index, or not. This leads to the following result:

Theorem 10.1. Let $\Gamma$ be a group from the following list of examples:

(H) hyperbolic groups that are not virtually cyclic,

(N-P) fundamental groups of closed Riemannian manifolds of non-positive sectional curvature of rank one and of dimension $\geq 2$,

(LAT) irreducible lattices in connected semisimple Lie groups with finite centre and rank $\geq 2$,

(MCG) mapping class groups of closed oriented surfaces of genus $\geq 1$,

(OUT) outer automorphism groups of free groups of rank $\geq 2$,

(END) groups with infinitely many ends.

Then no infinite subnormal subgroup of $\Gamma$ is presentable by a product. 
Recall that a subgroup $\Gamma_{0} \subset \Gamma$ is subnormal if there is a descending sequence of subgroups $\Gamma_{0} \subset \Gamma_{1} \subset \cdots \subset \Gamma_{k} \subset \Gamma_{k+1}=\Gamma$ such that $\Gamma_{i}$ is normal in $\Gamma_{i+1}$ for all $i \in\{0, \ldots, k\}$.

In order to give a quick and uniform proof for almost all the different cases we use the fact that all the groups in the theorem, except the lattices in (LAT), are in $\ell_{\text {reg }}$; compare the survey table in the Appendix. It was proved by Monod and Shalom [38], Proposition 7.4, that if $\Gamma$ is in $\ell_{\text {reg }}$, then so is every infinite normal subgroup. Theorem 10.1 then follows from Proposition 6.2 by induction on the length of the chain of subnormal subgroups. In the case of the lattices in (LAT), the Margulis normal subgroup theorem ([33], Chapter IV, [44], Chapter 8) implies that every infinite subnormal subgroup has finite index. The conclusion then follows from Proposition 8.1.

The case (N-P) in the theorem can be generalized further by considering CAT $(0)$ groups in the sense of [9]. Let $\Gamma$ be any discrete group that admits a proper, minimal, isometric action without fixed points at infinity on a proper, irreducible CAT(0)space $X$ with finite-dimensional boundary. If $X$ is not the real line, then no infinite subnormal subgroup of $\Gamma$ is presentable by products. This is implicit in a result of Caprace and Monod (Theorem 1.10 of [12]).

To put this extension into context, recall that an action of a group on a CAT(0)space is minimal if this space does not contain a non-empty invariant closed convex (proper) subspace. As in the Riemannian case, a CAT(0)-space is irreducible if it does not admit a non-trivial isometric splitting as a direct product. If a discrete group acts cocompactly via isometries on a proper CAT(0)-space $X$, then the boundary of $X$ is automatically finite-dimensional [27], Theorem C. Moreover, if a discrete group acts properly discontinuously, minimally, and cocompactly via isometries on a CAT(0)-space without Euclidean factors, then this action does not have any fixed points at infinity [2], Corollary 2.7.

10.2. Relations with geometric and with measurable group theory. The property of being or not being presentable by a product is not always shared by groups that are equivalent under one of the usual equivalence relations considered in geometric group theory.

Theorem 10.2. The property of being presentable by products is not invariant under quasi-isometries, under measure equivalence, or under orbit equivalence.

Proof. The isometry group of the polydisk $\mathbb{H}^{2} \times \mathbb{H}^{2}$ contains both reducible and irreducible cocompact lattices. The reducible ones are trivially presentable by products, whereas the irreducible ones are not presentable by products [28], Corollary 4.2.

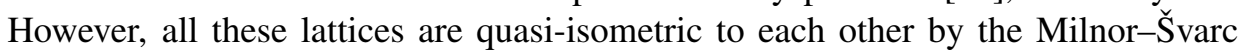
lemma. This shows that presentability by products is not a quasi-isometry invariant property. 
All infinite amenable groups admit orbit equivalent measure preserving free actions on standard Borel probability spaces [39]. Obviously, there are many amenable groups that are presentable by a product, for instance free Abelian groups of non-zero rank. However, there are also amenable groups that are not presentable by products; see Example 3.6. Thus, presentability by products is not invariant under orbit equivalence.

The examples mentioned in the previous paragraph also show that presentability by products is not invariant under measure equivalences (the class of groups that are measure equivalent to $\mathbb{Z}$ equals the class of all infinite countable amenable groups [14]).

In spite of Theorem 10.2, many of the obstructions against presentability by products that we have discussed in this paper have strong invariance properties under these equivalence relations. For example, the non-vanishing of the first $L^{2}$-Betti number is a quasi-isometry invariant [17], p. 19, p. 224, [6], p. 314. Moreover, Gaboriau proved that the vanishing of the first $L^{2}$-Betti number is an orbit equivalence invariant [16], Théorème 3.12, and a measure equivalence invariant [16], Théorème 6.3. Next, being expensive is an orbit equivalence invariant and a measure equivalence invariant for groups with fixed price as the cost of a group is defined in terms of its orbit relations [15], Propositions VI.5, VI.6. Finally, the non-vanishing of the second bounded cohomology with coefficients in the regular representation is a measure equivalence invariant [38], Corollary 7.6. Whether it is invariant under quasi-isometries seems to be unknown; see Problem J in Monod's 2006 ICM talk [36].

\section{Appendix: Overview of results}

Table 1 surveys the applicability of different criteria to proving that certain classes of groups are not presentable by products. The first column lists certain test classes of groups; of the other columns each corresponds to a way of concluding that groups are not presentable by products. The "ad hoc" column refers to the direct, low-tech, hands-on argument relying on information about the sizes of centralisers, including in particular the arguments about acentral extensions of Section 3. The other columns each use some high-brow theory.

The first test class of groups are the non-elementary hyperbolic groups, denoted $(\mathrm{H})$ in [28]. Non-elementary is the same thing as not virtually cyclic.

The second class are the mapping class groups of closed oriented surfaces of genus at least 3 . We omit genus 1 and 2 because they have special features that do not occur in high genus, e.g., they have non-trivial centres.

The third class are the outer automorphism groups of free groups $\operatorname{Out}\left(F_{n}\right)$, where we assume that $n \geq 3$. For $n=2$ one has $\operatorname{Out}\left(F_{2}\right)=\operatorname{GL}(2 ; \mathbb{Z})$.

The case of a free product $\Delta_{1} * \Delta_{2}$ with $\left|\Delta_{i}\right|>i$ is contained in the more general situation of a group with infinitely many ends considered separately here. However, 
Table 1. Overview of results

\begin{tabular}{|c|c|c|c|c|c|}
\hline properties of $\Gamma$ & ad hoc & $b_{1}^{(2)}(\Gamma)>0$ & $\varphi(\Gamma)>1$ & $\Gamma \in \mathcal{P}$ & $\Gamma \in \mathcal{C}_{\mathrm{reg}}$ \\
\hline hyperbolic (non-el.) & [28], Prop. 3.6 & - & -1 & -8 & [34], Thm. 3 \\
\hline MCG in genus $\geq 3$ & [28], Prop. 3.8 & - & -2 & [10], Thm. 2.2 & [19], Thm. 4.5 \\
\hline $\operatorname{Out}\left(F_{n}\right)$ for $n \geq 3$ & Prop. 8.3 & - & -3 & [10], Thm. 2.6 & [21], Cor. \\
\hline$|e(\Gamma)|=\infty$ & Prop. 9.1 & $\checkmark^{0}$ & $\checkmark$ & $-^{8}$ & [37], Cor. 7.9 \\
\hline$\Delta_{1} * \Delta_{2},\left|\Delta_{i}\right|>i$ & Example 3.4 & $\checkmark^{0}$ & $\checkmark$ & [22], Prop. 8 & [37], Cor. 7.9 \\
\hline$(\mathrm{N}-\mathrm{P})$, rank 1 & [28], Prop. 3.7 & - & -1 & $?$ & [20], Thm. 2 \\
\hline (N-P), irred., rk $\geq 2$ & [28], Thm. 4.1 & - & -4 & $?$ & $-^{5}$ \\
\hline $\operatorname{GL}(n, \mathbb{Z}), n \geq 3$ & Prop. 8.1 & - & -4 & $-{ }^{11}$ & $-^{5}$ \\
\hline Thompson $F$ & Prop. 7.1 & - & $-{ }^{10}$ & $?$ & $?$ \\
\hline$\Gamma \subset \mathrm{Sol}^{3}$ a lattice & Cor. 3.7 & - & -6 & -9 & -7 \\
\hline $\begin{array}{l}{ }^{0} \text { well known } \\
{ }^{1} \text { See Example } 4.1 \\
2 \text { See Proposition } \\
{ }^{3} \text { See Proposition } \\
{ }^{4} \text { Lattices in highe } \\
{ }^{5} \text { Lattices in almo } \\
{ }^{6} \text { See Lemma } 4.9 \\
{ }^{7} \text { The bounded col } \\
{ }^{8} \text { See Example } 5.2 \\
{ }^{9} \text { A non-trivial am } \\
{ }^{10} F \text { contains itsel } \\
{ }^{11} \mathrm{GL}(n, \mathbb{Z}) \text { has n }\end{array}$ & $\begin{array}{l}11 . \\
6 . \\
\text { rank Lie groups } \\
\text { simple higher r } \\
\text { [25], Propositi } \\
\text { omology of ame } \\
\text { nable group can } \\
\text { with finite index } \\
\text { n-trivial center, }\end{array}$ & $\begin{array}{l}\text { are cheap [15], } \\
\text { nk Lie groups a } \\
\text { n } 35.1 \text { (i). } \\
\text { able groups van } \\
\text { ot be } C^{*} \text {-simpl } \\
>1 \text {. } \\
\text { ad so cannot be }\end{array}$ & $\begin{array}{l}\text { Corollary VI. } \\
\text { e not in } \mathcal{C}_{\mathrm{reg}} \\
\text { ishes. } \\
C^{*} \text {-simple. }\end{array}$ & $\begin{array}{l}0 . \\
\text { 37], Theorem } 1 .\end{array}$ & \\
\hline
\end{tabular}

not all criteria that apply to free products generalize to groups with infinitely many ends.

By $(\mathrm{N}-\mathrm{P})$ we mean the class of fundamental groups of closed oriented manifolds of non-positive curvature, as considered in [28]. The results about this class can be extended to groups admitting suitable actions on CAT(0)-spaces; see Section 10.1.

For the Thompson group $F$ amenability seems to be an open question, but it is certainly not elementary amenable. The final example concerns the fundamental groups of 3-manifolds carrying the Thurston geometry $\mathrm{Sol}^{3}$. These are elementary amenable, and they show that none of the high-tech obstructions against presentability by products apply to arbitrary acentral extensions. 
Where a criterion does work for a class of groups, the corresponding entry in the table gives the earliest reference for a complete proof known to us. A horizontal line indicates that the criterion is not applicable; this is explained in the footnotes.

The checkmarks in the cost column come from Theorem 4.7. If a group can be shown not be presentable by products using the first $L^{2}$-Betti number, then one can also use the cost for this purpose. Conversely, if a group is cheap, then its first $L^{2}$ Betti number vanishes, and this explains the horizontal lines without footnotes in the $L^{2}$-Betti number column.

\section{References}

[1] M. Abért and N. Nikolov, Rank gradient, cost of groups and the rank versus Heegaard genus problem. J. Eur. Math. Soc. (JEMS) 14 (2012), 1657-1677. Zbl 06095894 MR 2966663

[2] S. Adams and W. Ballmann, Amenable isometry groups of Hadamard spaces. Math. Ann. 312 (1998), 183-195. Zbl 0913.53012 MR 1645958

[3] J. Amorós, M. Burger, K. Corlette, D. Kotschick, and D. Toledo, Fundamental groups of compact Kähler manifolds. Math. Surveys Monogr. 44, Amer. Math. Soc., Providence, R.I., 1996. Zbl 0849.32006 MR 1379330

[4] G. Baumslag and T. Taylor, The centre of groups with one defining relator. Math. Ann. 175 (1968), 315-319. Zbl 0157.34901 MR 0222144

[5] M. E. B. Bekka, M. Cowling, and P. de la Harpe, Simplicity of the reduced $C^{*}$-algebra of $\operatorname{PSL}(n, \mathbb{Z})$. Internat. Math. Res. Notices 1994 (1994), no. 7, 285-291. Zbl 0827.22002 MR 1283024

[6] M. E. B. Bekka and A. Valette, Group cohomology, harmonic functions and the first $L^{2}$-Betti number. Potential Anal. 6 (1997), 313-326. Zbl 0882.22013 MR 1452785

[7] M. Bestvina, K. Bromberg and K. Fujiwara, in preparation.

[8] M. Bestvina, M. Feighn, and M. Handel, Laminations, trees, and irreducible automorphisms of free groups. Geom. Funct. Anal. 7 (1997), 215-244; Erratum, ibid., 1143. Zbl 0884.57002 MR 1445386 MR 1487756

[9] M. R. Bridson and A. Haefliger, Metric spaces of non-positive curvature. Grundlehren Math. Wiss. 319, Springer-Verlag, Berlin 1999. Zbl 0988.53001 MR 1744486

[10] M. R. Bridson and P. de la Harpe, Mapping class groups and outer automorphism groups of free groups are $C^{*}$-simple. J. Funct. Anal. 212 (2004), 195-205. Zbl 1064.46052 MR 2065242

[11] J. W. Cannon, W. J. Floyd, and W. R. Parry, Introductory notes on Richard Thompson's groups. Enseign. Math. (2) 42 (1996), 215-256. Zbl 0880.20027 MR 1426438

[12] P.-E. Caprace and N. Monod, Isometry groups of non-positively curved spaces: structure theory. J. Topol. 2 (2009), 661-700. Zbl 1209.53060 MR 2574740

[13] H. Freudenthal, Über die Enden diskreter Räume und Gruppen. Comment. Math. Helv. 17 (1945), 1-38. Zbl 0060.40007 MR 0012214 
[14] A. Furman, Gromov's measure equivalence and rigidity of higher rank lattices. Ann. of Math. (2) 150 (1999), 1059-1081. Zbl 0943.22013 MR 1740986

[15] D. Gaboriau, Coût des relations d'équivalence et des groupes. Invent. Math 139 (2000), 41-98. Zbl 0939.28012 MR 1728876

[16] D. Gaboriau, Invariants $l^{2}$ de relations d'équivalence et de groupes. Publ. Math. Inst. Hautes Études Sci. 95 (2002), 93-150. Zbl 1022.37002 MR 1953191

[17] M. Gromov, Asymptotic invariants of infinite groups. Geometric group theory (Sussex, 1991), vol. 2. London Math. Soc. Lecture Note Ser. 182, Cambridge University Press, Cambridge 1993. Zbl 0841.20039 MR 1253544

[18] E. K. Grossman, On the residual finiteness of certain mapping class groups. J. London Math. Soc. (2) 9 (1974), 160-164. Zbl 0292.20032 MR 0405423

[19] U. Hamenstädt, Bounded cohomology and isometry groups of hyperbolic spaces. J. Eur. Math. Soc. (JEMS) 10 (2008), 315-349. Zbl 1139.22006 MR 2390326

[20] U. Hamenstädt, Isometry groups of proper CAT(0)-spaces of rank one. Groups Geom. Dyn. 6 (2012), 579-618. Zbl 06088871 MR 2961285

[21] U. Hamenstädt, Lines of minima in outer space. Preprint 2009. arXiv:0911.3620

[22] P. de la Harpe, Reduced $C^{*}$-algebras of discrete groups which are simple with a unique trace. In Operator algebras and their connections with topology and ergodic theory, Lecture Notes in Math. 1132, Springer-Verlag 1985, 230-253. Zbl 0575.46049 MR 0799571

[23] P. de la Harpe, On simplicity of reduced $C^{*}$-algebras of groups. Bull. London Math. Soc. 39 (2007), 1-26. Zbl 1123.22004 MR 2303514

[24] D. Johnson The structure of the Torelli group I: A finite set of generators for I. Ann. of Math. (2) 118 (1983), 423-442. Zbl 0549.57006 MR 0727699

[25] A. S. Kechris and B. D. Miller, Topics in orbit equivalence. Lecture Notes in Math. 1852, Springer-Verlag, Berlin 2004. Zbl 1058.37003 MR 2095154

[26] Y. Kida, The mapping class group from the viewpoint of measure equivalence theory. Mem. Amer. Math. Soc. 196 (2008), no. 16. Zbl 05498637 MR 2458794

[27] B. Kleiner, The local structure of length spaces with curvature bounded above. Math. Z. 231 (1999), 409-456. Zbl 0940.53024 MR 1704987

[28] D. Kotschick and C. Löh, Fundamental classes not representable by products. J. London Math. Soc. (2) 79 (2009), 545-561. Zbl 1168.53024 MR 2506686

[29] M. Lackenby, Expanders, rank and graphs of groups. Israel J. Math. 146 (2005), 357-370. Zbl 1066.22008 MR 2151608

[30] W. Lück, $L^{2}$-invariants: theory and applications to geometry and $K$-theory. Ergeb Math. Grenzgeb. (3) 44, Springer-Verlag, Berlin 2002. Zbl 1009.55001 MR 1926649

[31] M. Lustig, Conjugacy and centralizers for iwip automorphisms of free groups. In Geometric group theory, Trends Math., Birkhäuser, Basel 2007, 197-224. Zbl 1162.20020 MR 2395795

[32] W. Magnus, A. Karrass, and D. Solitar, Combinatorial group theory. 2nd ed., Dover Publications Inc., Mineola, NY, 2004. Zbl 1130.20307 MR 2109550

[33] G. A. Margulis, Discrete subgroups of semisimple Lie groups. Ergeb. Math. Grenzgeb. (3) 17, Springer-Verlag, Berlin 1991. Zbl 0732.22008 MR 1090825 
[34] I. Mineyev, N. Monod, and Y. Shalom, Ideal bicombings for hyperbolic groups and applications. Topology 43 (2004), 1319-1344. Zbl 1137.20033 MR 2081428

[35] N. Monod, Continuous bounded cohomology of locally compact groups. Lecture Notes in Math. 1758, Springer-Verlag, Berlin 2001. Zbl 0967.22006 MR 1840942

[36] N. Monod, An invitation to bounded cohomology. In Proc. Internat. Congr. Mathematicians, Vol. II, Eur. Math. Soc., Zürich 2006, 1183-1211. Zbl 1127.55002 MR 2275641

[37] N. Monod and Y. Shalom, Cocycle superrigidity and bounded cohomology for negatively curved spaces. J. Differential Geom. 67 (2004), 395-455. Zbl 1127.53035 MR 2153026

[38] N. Monod and Y. Shalom, Orbit equivalence rigidity and bounded cohomology. Ann. of Math. (2) 164 (2006), 825-878. Zbl 1129.37003 MR 2259246

[39] D. S. Ornstein and B. Weiss, Ergodic theory of amenable group actions. I. The Rohlin lemma. Bull. Amer. Math. Soc. (N.S.) 2 (1980), 161-164. Zbl 0427.28018 MR 0551753

[40] D. Osin, Rank gradient and torsion groups. Bull. London Math. Soc. 43 (2011), 10-16. Zbl 1245.20044 MR 2765544

[41] S. D. Promislow, A class of groups producing simple, unique trace, $\mathrm{C}^{*}$-algebras. Math. Proc. Cambridge Philos. Soc. 114 (1993), 223-233. Zbl 0799.46069 MR 1230130

[42] P. Scott, The geometries of 3-manifolds. Bull. London Math. Soc. 15 (1983), 401-487. Zbl 0561.57001 MR 0705527

[43] A. Thom, Low degree bounded cohomology and $L^{2}$-invariants for negatively curved groups. Groups Geom. Dyn. 3 (2009), 343-358. Zbl 1162.22021 MR 2486803

[44] R. J. Zimmer, Ergodic theory and semisimple groups. Monogr. Math. 81, Birkhäuser Verlag, Basel 1984. Zbl 0571.58015 MR 776417

Received March 18, 2010; revised September 04, 2011

D. Kotschick, Mathematisches Institut, LMU München, Theresienstr. 39, 80333 München, Germany

E-mail: dieter@member.ams.org

C. Löh, Fakultät für Mathematik, Universität Regensburg, 93040 Regensburg, Germany

E-mail: clara.loeh@mathematik.uni-regensburg.de 